\title{
Texture Classification by Combining Local Binary Pattern Features and a Self-Organizing Map
}

\author{
Markus Turtinen, Topi Mäenpää, and Matti Pietikäinen \\ Machine Vision Group, P.O.Box 4500, FIN-90014 University of Oulu, Finland \\ \{dillian, topiolli,mkp\}@ee.oulu.fi
}

\begin{abstract}
This paper deals with the combined use of Local Binary Pattern (LBP) features and a Self-Organizing Map (SOM) in texture classification. With this approach, the unsupervised learning and visualization capabilities of a SOM are utilized with highly efficient histogram-based texture features. In addition to the Euclidean distance normally used with a SOM, an information theoretic log-likelihood (cumlog) dissimilarity measure is also used for determining distances between feature histograms. The performance of the approach is empirically evaluated with two different data sets: (1) a texture-based visual inspection problem containing four very similar paper classes, and (2) classification of 24 different natural textures from the Outex database.
\end{abstract}

\section{Introduction}

A visual inspection and classification system is usually implemented with an ordinary parametric classifier based on supervised learning. In this kind of approach, a human controls the teaching procedure providing labels for the training samples and also choosing these samples. The main goal of the teacher is to reduce the average distance between the desired classification result and the current result. Human involvement makes this approach inconsistent and raises the risk for human errors. In addition, parametric classifiers often make erroneous assumptions about the structure of the data and can thus fail badly.

In unsupervised training, human involvement is not so crucial, because the training happens automatically according to the clustering properties of the training data. Recently, Niskanen et al. [1] introduced a method based on dimensionality reduction, visualization and classification with Self-Organizing Maps [2]. Fig. 1 shows the block diagram of a system based on non-supervised training. The main principle of the method is that similar data cluster close to each other in a low-dimensional space, allowing predictions to be made about the data by inspecting the clusters. The labeling of individual training samples is not required, and the role of the human is more like an observer than a teacher.

Texture plays an important role in many applications of machine vision, including visual inspection. Effective features form the basis for all texture related problems. Recently, the distributions of local binary patterns (LBP) have 


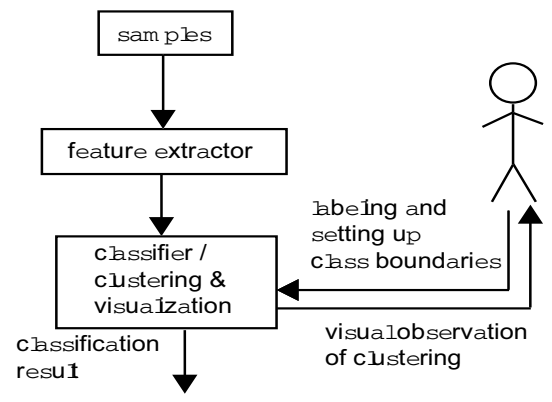

Fig. 1. Block diagram of a SOM-based non-supervised training

been successfully applied to various texture analysis problems and applications, suggesting that the LBP methodology is currently perhaps the most powerful approach to texture analysis [3].

Recently, we have made successful experiments with LBP and SOM in a paper characterization problem, where the characteristics and quality of the paper were inspected by analyzing the texture [4]. The LBP feature provided excellent discrimination of four different paper classes, while the SOM allowed visualization-based system training and fast classification. The combined use of LBP features with the SOM still leaves some open problems. In training a SOM and in classification, the Euclidean distance has been conventionally used. It was also used in our previous experiments. However, with histogram-based features like the LBP, an information theoretic distance measure is often preferred [5]. Also the performance of classification needs to be assessed more systematically, for example by using another classifier for comparison and additional data containing a larger number of texture classes.

In this paper, we consider the use of a log-likelihood (cumlog) distance measure with a SOM and compare its performance to that obtained with the Euclidean measure using the same paper characterization data as in [4]. The cumlog distance measure has been often used with LBP [3]. For comparison, a $k$-NN classifier is also used with the LBP features. The performance of the approach presented is also assessed with another data set containing 24 texture classes from the Outex database [6]

\section{Using Local Binary Pattern Features with Self-Organizing Map}

\section{Local Binary Pattern features}

LBP is a gray-scale invariant texture primitive statistic [7]. Fig. 2 shows how LBP is calculated. For each pixel in an image, a binary code is produced by thresholding its neighborhood with the value of the center pixel. A histogram 
is then constructed to collect up the occurrences of different binary patterns representing different types of curved edges, spots, flat areas etc. The bins of the histogram formed are independent and even adjacent bins might represent totally different patterns. In addition, the sizes of the bins can vary greatly.

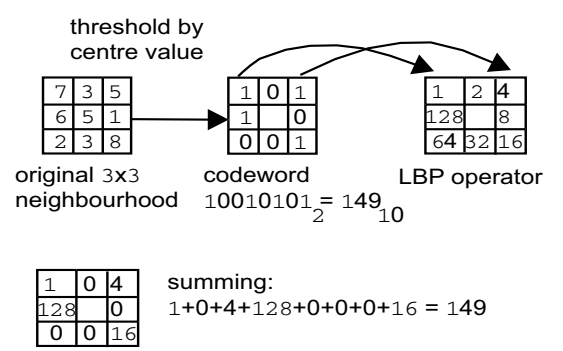

Fig. 2. Computation of LBP feature

The basic 8-bit version of the LBP operator considers only eight neighbors of each pixel at distance one, but the definition can be extended to include all circular neighborhoods with any number of pixels [3]. This makes it possible to use larger and more discriminating histograms.

Special care must be taken when distributions are used as features for a classification system. The size of an LBP histogram is dependent on the size of the image, which must be taken into account by normalizing the feature vectors. This is easiest to achieve by dividing all the members of the vector by the number of pixels in the image. Of course, when the size of the image remains constant, there is no need for such normalization.

In the classification phase, the dissimilarity between histograms needs to be determined. Many well-known information-theoretic dissimilarity measures, like the Kullback-Leibler divergence and the Jeffrey-divergence can be utilized for that purpose [5]. With LBP distribution, the log-likelihood statistic based on Kullback's minimum cross-entropy has been usually used [3, 7]. This cumlog statistic is calculated as

$$
L(S, M)=\sum_{b=1}^{B} S_{b} \log M_{b},
$$

where $S$ and $M$ are the sample and model distributions, $B$ is the number of bins and $S_{b}$ and $M_{b}$ correspond to the sample and model probabilities at bin $b$ [3]. In addition to these kinds of dissimilarity measures, which are often used when comparing distributions, the simple Euclidean distance normally used with SOM could also be used for this purpose. 


\section{The Self-Organizing Map}

The Self-Organizing Map is a non-supervised learning and clustering method based on artificial neural networks [2]. Like the other neural networks, a SOM also has a finite number of neurons, nodes that process the data. Nodes are organized, in the two-dimensional case, on a previously defined regular grid. When multi-dimensional data is fed to the SOM for training purposes, each of its nodes is assigned specific vector values representing the training feature vectors. Multi-dimensional feature vectors of nodes form a non-linear projection to the low-dimensional space and make it possible to visualize the data.

The training algorithm is run iteratively and in a non-supervised fashion. In the beginning, the nodes of the SOM are initialized, which can be done, for example, by randomly selecting data from the training set, and placing them to the nodes. After initialization, the algorithm compares the training vectors to the vectors of the nodes and tries to find the node whose vector is nearest to the training vector. Then the feature vectors of the selected node and its neighbors are adjusted for matching better with the training vector. So the algorithm affects not only to the selected node, but also its neighbors and causes hierarchical organization of the neural network and data. By labeling the nodes with the training data, the SOM can be used as a classifier.

\section{Combining $L B P$ and $S O M$}

LBP provides a varying number of bins depending on the specific LBP operator used [3]. For example, the size of the basic 8-bit $\operatorname{LBP}_{8,1}$ histogram is 256 bins while it can be more than 16 million for a multi-resolution $\operatorname{LBP}_{8,1+16,2+24,3}$. Basically the length of the distribution can vary greatly, but in reality it must be restricted according to the other parts of the system. For example, the timing limits and calculation capacity must be considered: the memory requirements and the time elapsed by training and classification are in direct relationship to the size of the feature vectors and the amount of data. Fortunately, recent powerful extensions of the LBP based on 'uniform' patterns allow us to reduce the size of the histograms dramatically [3].

When using a given LBP operator for input images of a fixed size, the members of the feature vector do not need to be necessarily normalized to make them commensurate. This is required only if the sizes of the histograms can vary. Usually this is done by normalizing the sum of bins to 1 . Another popular normalization scheme is to set the mean of the feature vector to 0 and variance to 1 . This causes negative values for the vector, making it impossible to use with a log-likelihood distance measure. Normalizing the sum of the histogram bins to 1 causes the sizes of the individual bins to vary between 0 and 1 . This can be taken into account in the log-likelihood calculations by making the sum of Eq. 1 negative.

After the training phase, the codebook vectors of the SOM (values of trained nodes) are proportional to the features of the training data, and each node can be 
considered like the feature vector extracted from a training sample. If the created SOM is used as a classifier, or for some other purpose by using separate data as input, the new data must be brought to the same form as the training data. For example, the new data must be normalized with the same normalization parameters.

Fig. 3 illustrates the idea of clustering based classification in paper characterization, but the principle is same for any classification problem. Classification with a SOM is simple: samples can be compared directly to the nodes of the SOM and only the closest node needs to be found. To achieve quantitative results with a SOM classifier, a pre-labeled SOM must be used. Labeling is usually performed with supervision, which causes the SOM classifier to be not fully non-supervised. By inspecting just the clustering of data, based on the samples appearances, predictions on class boundaries can be visually made.

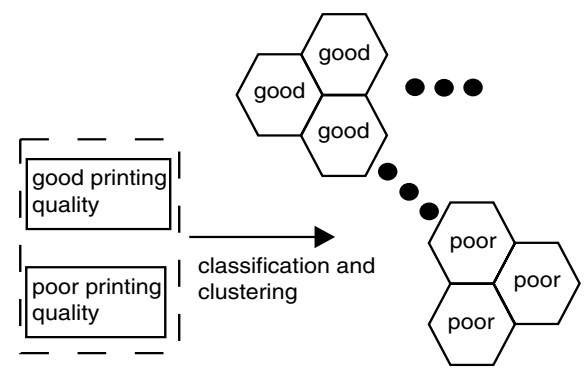

Fig. 3. SOM-based clustering and classification

\section{Experiments}

The performance of SOM-based classification with the LBP features was analyzed with two different data sets. The cumlog distance measure was applied and the result achieved by it was compared to those obtained with the Euclidean measure. Also the speed and accuracy of the SOM-based classifier were compared to those of a $k$-NN classifier, which is generally considered to be the most accurate and closest to the Bayesian model of non-parametric classifiers $[8]$.

\subsection{Experiments with paper data}

In [4], we made paper characterization experiments with different texture features. LBP outperformed, by a clear margin, previously used methods in paper inspection, including those based on gray-level co-occurrences, Fourier analysis 
and specific perimeter. With four classes, the basic $\mathrm{LBP}_{8,1}$ combined with the SOM-based classification gave $98.4 \%$ classification accuracy, with multiresolution and uniform $\operatorname{LBP}_{8,1+16,2+24,3}$ the accuracy was $99.8 \%$, while it was 75.7 $\%$ with FFT, $68.0 \%$ with co-occurrence and $56.1 \%$ with specific perimeter, respectively.

The experiments described above were carried out by using the Euclidean distance measure for the SOM in training and classification. The data set consisted of 1004 pieces of 8-bit grey scale images. All the images were of size $760 * 570$ pixels and the total number of classes was 4 . A hold-out classification test with approximately equally sized and randomly divided training (501 samples) and test (503 samples) sets was performed. The size of the classification SOM was set to $10^{*} 8$ nodes, which is small enough to reach a high-speed classification rate and large enough to achieve a good discrimination between classes of used data.

Table 1 summarizes the classification results and execution times, including our new experiments with the cumlog distance and $k$-NN $(k=3)$ classifier (accuracy [\%] and classification time [ms]) achieved by both classifiers using both basic and multiresolution LBP. Times are calculated as a mean of several runs made on a $900 \mathrm{MHz}$ Sun Sparc. Feature extraction itself took $21 \mathrm{~ms}$ when the basic 8-bit LBP was used and $6.90 \mathrm{~s}$ with the multiresolution version of LBP.

\begin{tabular}{|c|c|c|c|c|}
\hline Classification Scheme & Cumlog [\%] & Cumlog Ct [ms] & Euclidean [\%] & Euclidean Ct [ms] \\
\hline Basic LBP + SOM & 98.8 & 8.2 & 98.4 & 2.5 \\
MR-LBP + SOM & 98.6 & 31.3 & 99.8 & 3.7 \\
\hline Basic LBP + k-NN & 99.8 & 51.3 & 99.8 & 4.4 \\
MR-LBP + $k$-NN & 99.8 & 181.2 & 99.8 & 8.2 \\
\hline
\end{tabular}

Table 1. Paper characterization results

It can be noticed that the SOM-based classification is much faster than the $k$-NN classifier. Logarithm calculations in the cumlog dissimilarity measure take a lot of time, but they can be partly optimized by using pre-calculated look-up tables. It should also be noticed that the times elapsed in the different schemes are not directly linear dependent on the size of the vector and maximum amount of comparisons. This is because the distance calculation is cancelled if the current minimum distance is exceeded to save unnecessary comparisons.

The classification performances are very similar for both distance measures. With the $k$-NN classifier both dissimilarity measures worked well and gave the same results. With the SOM classifier and basic LBP, the advantage of the cumlog was 0.4 percentage units, but with multiresolution LBP, the cumlog lost 1.2 percentage units and gave even worse results than the basic LBP.

The data set used turned out to be easy for LBP. By using such effective features with such a small set, it is hard to find reliable differences in performance between classifiers and dissimilarity measures. The differences achieved between dissimilarity measures and SOM-based classifiers are so small that they could be caused by the nature of random procedures in the SOM's initialization. But 
in addition to the randomness, also the numerical loss of data when normalizing the data between 0 and 1 could cause some decrease in accuracy, especially when calculating the cumlog distance and taking logarithms of such a floating number data is constantly needed. To assess the performance of the SOM-based classifier using LBP histograms and a cumlog distance measure, a more advanced test with different data sets had to be made.

\subsection{Experiments with Outex data}

In the second experiment, a set of 24 textures from the Outex database [6] was used. At the Outex site, this test set has the test suite ID Outex_TC_000002. The suite is produced by taking the maximum possible number of non-overlapping $32 \times 32$ pixel subimages from each gray-scale image. This results in a total number of 8832 images, and 368 samples per texture. These are randomly divided into two even sets, one for training and the other for testing. The random division is performed 100 times. As a classification result, the mean of the classification accuracy over the 100 trials is reported.

Similar tests to those in Section 3.1 were made, and Table 2 shows the results. In the SOM-based classification, the size of the SOM was set to $25 * 25$ nodes and the $k$-NN classification was made with $k$-value 3 . Feature extraction took $0.09 \mathrm{~ms}$ with the basic LBP and $3.34 \mathrm{~s}$ with the multiresolution operator.

\begin{tabular}{|c|c|c|c|c|}
\hline Classification Scheme & Cumlog [\%] & Cumlog Ct [ms] & Euclidean [\%] & Euclidean Ct [ms] \\
\hline Basic LBP + SOM & 88.4 & 75.1 & 81.7 & 8.2 \\
MR-LBP + SOM & 88.4 & 263.3 & 81.8 & 14.4 \\
\hline Basic LBP + $k$-NN & 88.6 & 549.2 & 88.1 & 58.6 \\
MR-LBP + $k$-NN & 91.1 & 657.3 & 90.3 & 71.6 \\
\hline
\end{tabular}

Table 2. Outex classification results

We can see that the cumlog distance now gives greater accuracy than the Euclidean distance. Especially with the SOM-based approach and the basic LBP, the improvement is noticeable and the results are comparable to those of $k$ $\mathrm{NN}$ classification. In $k$-NN classification the multiresolution $\mathrm{LBP}_{8,1+16,2+24,3}$ performs slightly better than the basic LBP, but this result is not necessarily statistically reliable, because the size of the images is so small ( $32 * 32$ pixels). The multiresolution LBP histogram is obtained by concatenating histograms of uniform LBP patterns at different distances into a single histogram. The sizes of the histograms used were 59,243 and 555 bins obtained at radii 1, 2 and 3, respectively. The images were $32 * 32$ pixels predisposing at least the largest 555 bins histograms to statistical noise.

With a SOM-based approach, the multiresolution LBP performed almost equally well than the basic LBP, while in $k$-NN classification it was better. This can be partly caused by the statistical instability of the feature, but also the numerical loss of data in the iterative process is significant. The histograms 
contain too much zero-valued data, and while iterating the training and updating processes thousands of times, the numerical loss tends to accumulate and affect the values of the node vectors.

\section{Conclusions}

This paper considered problems of combining Local Binary Pattern texture features with a SOM-based classification. Very good performance was obtained in two different classification problems. It was shown that a log-likelihood distance measure can be successfully used instead of the Euclidean distance normally used with the SOM. The cumlog measure performed significantly better when the number of classes grew. This provides us with great potential for using LBP and SOM in more challenging texture analysis problems than the 4-class problem used in our earlier studies. The unsupervised learning, visualization and high-speed classification capabilities of the SOM combined with the excellent discrimination capability and the computational simplicity of the LBP offer significant potential for new industrial applications. We have plans to use the approach presented in a realistic paper characterization application, where the number of paper grades can be several dozen.

\section{References}

1. Niskanen, M., Silvén, O., Kauppinen, H.: Color and texture based wood inspection with non-supervised clustering. In: SCIA 2001 - 12th Scandinavian Conference on Image Analysis, Bergen, Norway (2001)

2. Kohonen, T.: Self-Organizing Maps. Springer-Verlag, Berlin, Germany (1997)

3. Ojala, T., Pietikäinen, M., Mäenpää, T.: Multiresolution gray-scale and rotation invariant texture classification with local binary patterns. IEEE Transactions on Pattern Analysis and Machine Intelligence 24 (2002) 971-987

4. Turtinen, M., Pietikäinen, M., Silvén, O., Mäenpää, T., Niskanen, M.: Texture-based paper characterization using non-supervised clustering. In: Accepted to QCAV 2003 - 6th International Conference on Quality Control by Artificial Vision, Gatlinburg, Tennessee, USA (2003)

5. Puzicha, J., Buhmann, J., Rubner, Y., Tomasi, C.: Empirical evaluation of dissimilarity for color and texture. In: The 7th IEEE International Conference on Computer Vision, Kerkyra, Greece (1999)

6. Ojala, T., Mäenpää, T., Pietikäinen, M., Viertola, J., Kyllönen, J., Huovinen, S.: Outex - new framework for empirical evaluation of texture analysis algorithms. In: Proc. 16th International Conference on Pattern Recognition. Volume 1., Quebec, Canada (2002) 701-706

7. Ojala, T., Pietikäinen, M., Harwood, D.: A comparative study of texture measures with classification based on feature distributions. Pattern Recognition 29 (1996) $51-59$

8. Duda, R., Hart, P., Stork, D.: Pattern Classification. John Wiley \& Sons Inc. (2001) 2 ed. 